\title{
Health-related quality of life evaluation in patients with non- surgical benign tracheal stenosis
}

\author{
Benoit Jacques Bibas ${ }^{1}$, Paulo Francisco Guerreiro Cardoso ${ }^{1}$, Michele Salati ${ }^{2}$, Helio Minamoto ${ }^{1}$, Mauro \\ Federico Luiz Tamagno ${ }^{1}$, Ricardo Mingarini Terra ${ }^{1}$, Paulo Manuel Pêgo-Fernandes ${ }^{1}$ \\ ${ }^{1}$ Division of Thoracic Surgery, Heart Institute (InCor), Hospital das Clinicas da Faculdade de Medicina da Universidade de São Paulo. São Paulo, \\ Brazil; ${ }^{2}$ Unit of Thoracic Surgery, AOU Ospedali Riuniti di Ancona, Ancona, Italy \\ Contributions: (I) Conception and design: BJ Bibas, PF Cardoso, H Minamoto, MF Tamagno; (II) Administrative support: RM Terra, PM Pêgo- \\ Fernandes; (III) Provision of study materials or patients: BJ Bibas, PF Cardoso, H Minamoto, MF Tamagno; (IV) Collection and assembly of data: BJ \\ Bibas, MF Tamagno; (V) Data analysis and interpretation: All authors; (VI) Manuscript writing: All authors; (VII) Final approval of manuscript: All \\ authors. \\ Correspondence to: Benoit Jacques Bibas. Rua Dr. Enéas de Carvalho Aguiar 44, bloco 2, 2o andar, sala 9, Cerqueira Cezar, São Paulo, SP 05403-000, \\ Brazil. Email: benoit.bibas@hc.fm.usp.br.
}

\begin{abstract}
Background: The primary objective of the study was to evaluate the health-related quality of life (HRQL) of patients with benign post-intubation tracheal stenosis considered as unfit for surgical treatment. Secondary objectives were: (I) to determine if clinical variables (gender, age, total treatment time, and type of tracheal device) could influence HRQL and (II) to compare the results with a normal standardized population.

Methods: Prospective study between August-2014 and December-2016 including patients with tracheal stenosis treated with silicone stents, T-Tubes or tracheostomy. Candidates to airway resection and reconstruction were excluded from the analysis. HRQL was assessed with the SF-36 Health Questionnaire. Backward stepwise regression model analyzed the influence of clinical variables on the SF-36 domains and component summaries. Norm-based results were compared with normative data. Alpha error was $5 \%$.

Results: Ninety-three patients $(62 \mathrm{M} / 31 \mathrm{~F}$; mean age $38 \pm 14$ years) were included. Mean overall HRQL in all 8 SF-36 domains was poor. Lowest scores were in the role physical (mean 31.7 \pm 38 ), bodily pain (mean 39.2 \pm 35 ), and role emotional domain (mean 48.7 \pm 40 ). The physical component summary (PCS) was more affected than the mental $(\mathrm{P}<0.001)$. Norm-based results indicated that most domains and both physical and mental summaries were below the mean for the USA normative population. Total stenting time significantly influenced the physical $(\mathrm{P}=0.001)$ and mental component summaries $(\mathrm{P}=0.001)$.

Conclusions: Quality of life of patients with benign tracheal stenosis is severely impaired, and normbased results indicate that the HRQL is below normative data. Physical and mental discomfort seems to be attenuated by total treatment time.
\end{abstract}

Keywords: Tracheal stenosis; health-related quality of life (HRQL); tracheostomy

Submitted Feb 26, 2018. Accepted for publication Jul 03, 2018.

doi: $10.21037 /$ jtd.2018.07.80

View this article at: http://dx.doi.org/10.21037/jtd.2018.07.80

\section{Introduction}

Benign tracheal stenosis may follow prolonged orotracheal intubation, and it is estimated to occur in $1.8 \%$ to $12 \%$ of long-term invasively ventilated patients (1). Patients with tracheal or laryngotracheal stenosis often experience dyspnea, stridor, phonation ablation, dysphonia and dysphagia. Definitive treatment includes surgical resection and primary reconstruction with end-to-end anastomosis $(2,3)$. However, in patients considered unfit for surgery for technical reasons or with comorbidities, airway patency is ensured by means of prolonged airway stenting with or without tracheostomy (3). 
Health-related quality of life (HRQL) is a multidimensional concept reflecting the overall condition of the physical and mental welfare of the individual, which is a consequence not only of the disease but also of the family and social conditions forming the environment of the patient $(4,5)$. The Short Form 36 Health Questionnaire (SF-36) is a generic questionnaire, originally developed in the United States and translated and adapted for use in various countries according to the protocol of the International Quality of Life Assessment Project $(4,5)$. The impact of tracheal devices in the HRQL of patients with benign tracheal stenosis is unknown. It also remains unclear whether patients develop tolerance to the devices over time.

Therefore, the primary objective of this study was to evaluate the overall HRQL of patients with benign postintubation tracheal stenosis that were unfit for definitive surgical treatment. Secondary objectives were: (I) to determine if clinical variables (gender, age, total treatment time, and type of tracheal device) could influence the overall HRQL and to (II) compare our norm-based results with a standardized population.

\section{Methods}

Prospective study that enrolled patients with benign postintubation tracheal or laryngo-tracheal stenosis treated from August 2014 to December 2016. This institution is a national academic referral center for tracheal and airway diseases. The study was approved by the local Institutional Review Board (Cappesq \#719897). Date of approval: February $12^{\text {th }}, 2014$.

\section{Patient selection and enrollment}

We included patients treated with Montgomery T-tubes, endotracheal silicone stents or tracheostomy. Inclusion criteria were age between 18 and 65 years, willingness to participate in the study and sign an informed consent, and ability to fill out a questionnaire. Patients who were candidates to a definitive tracheal or laryngo-tracheal resection and reconstruction were excluded from the present analysis, as well as individuals with previous tracheal/laryngotracheal resection. In order to obtain a more homogenous population and to ensure an evaluation of the sole impact of stenting in the HRQL, we also excluded patients with chronic disabling diseases that could interfere with the HRQL evaluation, such as active malignant disease, advanced COPD (GOLD II-IV), heart failure (NYHA grades II-IV) and chronic renal failure on renal replacement therapy.

At our institution, contraindications for definitive surgical treatment of tracheal stenosis are: (I) stenosis longer than $5 \mathrm{~cm}$; (II) severe cognitive impairment; (III) severe cardiovascular disease; (IV) ongoing mechanical ventilation, and $(\mathrm{V})$ need for other forthcoming surgical procedures requiring tracheal intubation and general anesthesia. Type I diabetes, chronic renal failure, and active inflammation of the tracheal mucosa are considered relative contraindications to tracheal resection.

\section{Management}

All patients were evaluated with a computed tomography of the neck and chest, and a flexible bronchoscopy prior to treatment. The selection of non-surgical treatment followed the institutional guidelines approved by the local ethics committee, as follows:

* Montgomery T-Tube: patients with a pre-existing tracheostomy were treated with silicone T-tubes because it is a simple, safe and inexpensive method for tracheal stenting, as well as for restoring nasal airflow and speech (6);

* Endotracheal silicone stents: patients without a tracheostomy were treated with balloon dilation, or dilation of the stenosis followed by the placement of an endotracheal silicone stent that also restores nasal airflow, and avoids scarring and showing of a device in the neck region (2);

* Tracheostomy: approximately $80 \%$ of patients referred to our institution with tracheal stenosis have a tracheostomy. Since it is our policy to maintain the tracheostomy as little as possible, the patients with a contraindication to surgical treatment will be offered a silicone T-Tube as a palliative or definitive measure (3). Patients with tracheostomy in this study were evaluated while awaiting T-Tube placement.

\section{Data collection}

HRQL was evaluated using the Short Form-36 Health Survey version 2 (SF-36v2 ${ }^{\mathrm{TM}}$, Medical Outcomes Trust, Boston, MA, USA), with Portuguese translation validated in Brazil (7). The patient filled out the form in a private room of the outpatient unit after a brief explanation of its contents. The questionnaires were applied 1 month after 
stent placement (T-Tubes and Dumon Stents), or during the first consultation at the outpatient clinic, for individuals with tracheostomy. A physician was available to answer the patient's queries.

SF-36.v2 is a generic questionnaire for evaluating HRQL through a 36-item short form survey. It assesses eight different health domains as perceived by patients (physical functioning; role physical; bodily pain; general health; vitality; social functioning; role emotional; mental health). Each domain is transformed into a 0 to 100 scale. The lower the score, the more disability the patient shows. Therefore, a score of zero indicates maximum disability whereas a score of 100 is equivalent to no disability. Using scores standardized to norms and weighted averages for each dimension, it is possible to obtain two cumulative domains: The physical component summary (PCS) and the mental component summary (MCS) scores on a standard scale $(4,5)$.

Results are also analyzed as norm-based scores and compared to normal general populations. In norm-based scores, each domain is scored to have the same average [50] and the same standard deviation [10]. Therefore, for all health dimensions and component scales, any score less than 50 falls below the general population mean and each point represents $1 / 10^{\text {th }}$ of a standard deviation. This allows a direct comparison of the measures among different populations. For the purpose of this study, we used the 1998 USA population as comparison, as it is the most widely used reference.

\section{Clinical variables}

Age, gender, total treatment time (months) and the type of tracheal device were considered as possible predictors for HRQL changes.

Total treatment time is defined as the total amount of time that an individual has used the tracheal device, regardless of how many times this device has been changed.

\section{Statistical analysis}

Continuous variables are presented as means and standard deviation. The Shapiro-Wilk test was used for the assessment of normality. Categorical variables are presented as absolute numbers and percentage. SF-36 domain scores are reported as means and standard deviation. A backward stepwise regression model analyzed the influence of clinical variables (age, gender, total treatment time and type of tracheal device) on the SF-36 domains. SF-36 Norm-based scores were compared with the 1998 normative data for the United States of America Population. Alpha error was set at $5 \%$.

The present study was carried out taking into account the ethical principles of Good Clinical Practice as stated by the Imperial College Clinical Research Governance Office (http://www.imperial.ac.uk/clinicalresearchoffice). The researchers followed the ethical rules for the medical research involving humans, reported within the Declaration of Helsinki by the World Medical Association (8). All the patients involved in this analysis gave their informed consent. The study was approved by the local Institutional Review Board (Cappesq \#719897).

\section{Results}

Ninety-three patients were included $(62 \mathrm{M} / 31 \mathrm{~F})$, and the mean age was $38 \pm 14$ years. The flowchart of the study is depicted in Figure 1. Baseline characteristics of the patients are described in Table 1. Overall results with the SF-36 scores are shown in Table 2. The lowest scores were in the role physical domain (mean $31.7 \pm 38$ ), bodily pain (mean 39.2 \pm 35 ), and role emotional domain (mean 48.7 \pm 40 ). The PCS was more severely affected than the MCS $(\mathrm{P}<0.001 ; 95 \%$ CI: -14.5 to -7.3$)$.

The SF-36 norm-based results for the entire cohort were compared with the data of the United States Population and are available in Figure 2. The data depicts the norm-based scores for each domain and for their summaries (physical and mental). Only one domain (Vitality) reached the mean of the USA population. Most domains (Physical Function, General Health, Social Function and Mental Health) are 1 standard deviation below the mean, or 2 standard deviations below the mean (role physical, bodily pain, role emotional). Both summaries, physical and mental, are 1 standard deviation below the mean of the USA population. The regression analyzed the influence of clinical variables on the mental and physical component summaries of the SF-36 scores. Total stenting time was the only factor that affected the overall quality of life. It significantly influenced both the physical and mental component summaries. As each month passes, both the mental and the physical component summaries are scores are increased. Thus, a 1-month increase in total stenting time induces a 1.61 fold increase the in the MCS of the SF-36 HRQL and a 1.73 fold increase in the physical component (Tables 3,4). 


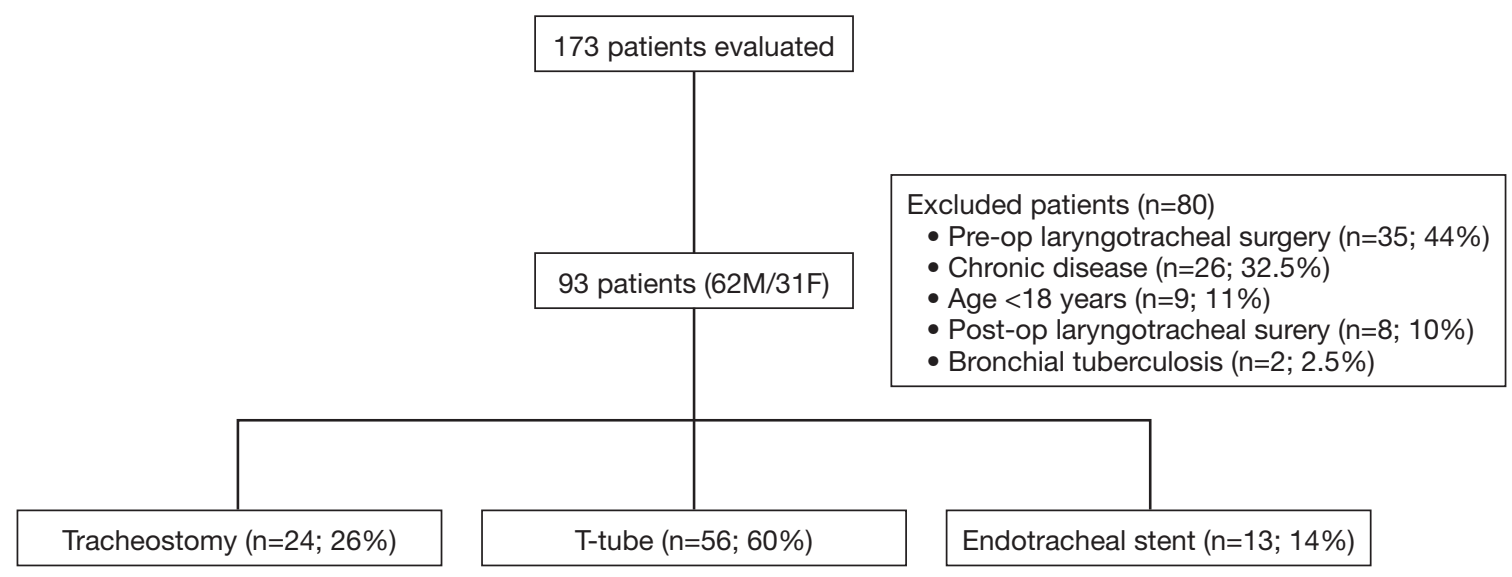

Figure 1 Flowchart of the study.

Table 1 Demographics

\begin{tabular}{lc}
\hline Characteristics & Data \\
\hline Sex , n (\%) & $62(67 \%)$ \\
Male & $31(33 \%)$ \\
Female & $38 \pm 14[18-65]$ \\
Age, years & $4.4 \pm 3.5$ \\
Total treatment time, months & \\
Type of treatment & $56(60 \%)$ \\
T tube & $24(26 \%)$ \\
Tracheostomy & $13(14 \%)$ \\
Stent & \\
\hline
\end{tabular}

Table 2 Overall results with the SF-36 survey

\begin{tabular}{lc}
\hline SF-36 domain & Mean \pm SD \\
\hline Physical function & $63.2 \pm 26$ \\
Role physical & $31.7 \pm 38$ \\
Bodily pain & $39.2 \pm 35$ \\
General health & $56.3 \pm 23$ \\
Vitality & $59 \pm 22$ \\
Social function & $62.7 \pm 26$ \\
Role emotional & $48.7 \pm 40$ \\
Mental health & $63.7 \pm 20$ \\
PCS & $47.6 \pm 24$ \\
MCS & $58.5 \pm 22$ \\
\hline
\end{tabular}

$\mathrm{SD}$, standard deviation; PCS, physical component summary; MCS, mental component summary.

\section{Discussion}

Airway resection with primary reconstruction by endto-end anastomosis is the definitive treatment for benign post-intubation tracheal stenosis $(2,3,9-12)$. However, a variety of factors such as long-segment stenosis, significant comorbidities, failed previous repair, and patient refusal to undergo surgery, may dictate nonsurgical management $(2,3)$. Our patient population is rather young. Most of our patients with post-intubation tracheal stenosis are trauma victims of motorcycle accidents, with head injuries that are often submitted to tracheostomy at the primary care center before they are referred to our center. At present, $80 \%$ of our patients with subglottic or tracheal stenosis have a tracheostomy when they are seen at the airway surgery outpatient clinic of our institution $(2,3)$. This reflects the high number of $\mathrm{T}$ tubes in our patient population who awaits definitive surgical treatment for the tracheal stenosis. A smaller portion of these patients have a long segment tracheal stenosis and are stented as a definitive treatment. Furthermore, in our experience, individuals with stenosis over $4 \mathrm{~cm}$ that were submitted to tracheal resection have a higher chance of overall complications (OR 5.91; $\mathrm{P}=0.001$ ) (2). Thus, we have a strict policy regarding surgical treatment form stenosis over $4 \mathrm{~cm}$.

In the present study we showed that individuals with benign post-intubation tracheal stenosis treated with stents or tracheostomy have an overall poor HRQL. Of the eight SF-36 domains, only one (Vitality) reached the mean of the USA population. Moreover, both the physical and the MCS were deeply affected, illustrating how much the HRQL is impaired in this group of patients. The PCS was more severely affected than the MCS $(\mathrm{P}<0.001)$. Our 

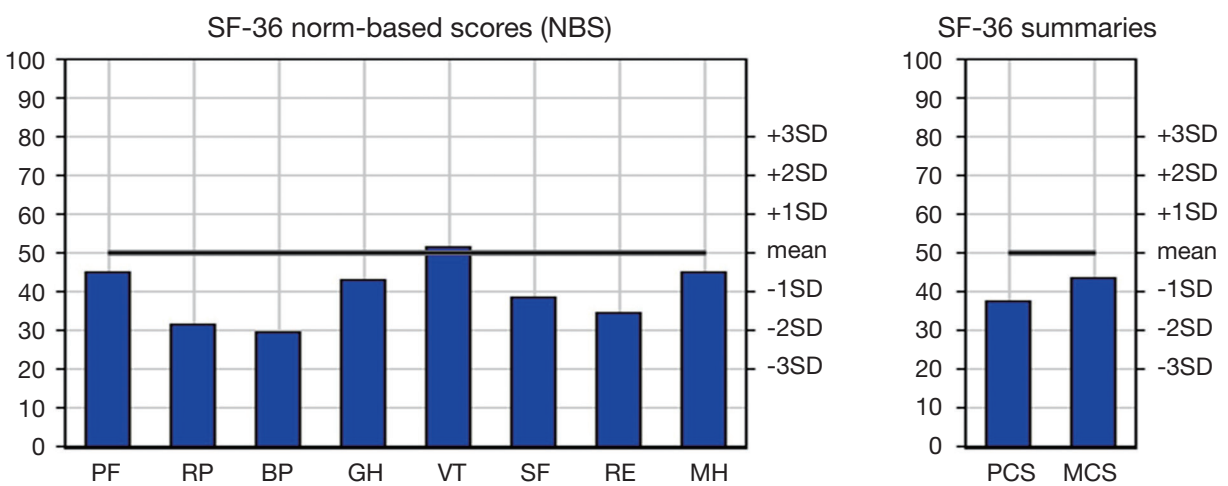

Figure 2 Norm-based scores of the SF-36 health survey for the entire cohort. PF, physical function; RP, role physical; BP, bodily pain; GH, general health; VT, vitality; SF, social function; RE, role emotional; $\mathrm{MH}$, mental health.

Table 3 Multivariate regression analysis for the MCS

\begin{tabular}{lccc}
\hline Parameter & Coefficient & $95 \% \mathrm{Cl}$ & $\mathrm{P}$ \\
\hline Age, years & -0.29 & -0.62 to 0.02 & 0.07 \\
$\begin{array}{l}\text { Total treatment } \\
\text { time, months }\end{array}$ & 1.61 & 0.30 to 2.91 & 0.016 \\
$\begin{array}{l}\text { Type of tracheal } \\
\text { device }\end{array}$ & 5.82 & -1.5 to 13.2 & 0.12 \\
Gender & -2.44 & -11.9 to 7.0 & 0.61 \\
\hline
\end{tabular}

MCS, mental component summary.

Table 4 Multivariate regression analysis for the PCS

\begin{tabular}{lccc}
\hline Parameter & Coefficient & $95 \% \mathrm{Cl}$ & $\mathrm{P}$ \\
\hline Age, years & -0.23 & -0.57 to 0.1 & 0.16 \\
$\begin{array}{l}\text { Total treatment time, } \\
\text { months }\end{array}$ & 1.76 & 0.38 to 3.14 & 0.013 \\
$\begin{array}{l}\text { Type of tracheal device } \\
\text { Gender }\end{array}$ & 7.44 & -0.32 to 15.2 & 0.06 \\
\hline
\end{tabular}

PCS, physical component summary.

results also indicate that the total treatment time could play an important role in the HRQL. It significantly influenced both mental and the physical component summaries.

It would be reasonable to assume that individuals treated with endotracheal devices could have a better HRQL than patients with T-tubes or tracheostomy but our data did not support that assumption. In fact, in the regression model used, the type of stent did not significantly affect either of the domains. Nonetheless, this was not the primary endpoint of our study. Thus, a prospective study with a larger number of patients, longer observation period and multiple serial surveys will be necessary to clarify this issue.

Few studies have addressed the HRQL of the individual with tracheal stenosis and stents. Gilony et al. (13) conducted a prospective trial including patients with benign tracheal stenosis. The study divided the patients into three groups: (I) patients with a tracheostomy awaiting laryngotracheal reconstruction; (II) decannulated patients after tracheal surgery; (III) control group without tracheal disease. Patients filled the Satisfaction with Life Scale, and the Body Cathexis Scale that focuses on the analysis of general well-being and the satisfaction from one's different body organs and functions, respectively. Results showed that patients with tracheostomy had significantly reduced scores if compared with the control group. Decannulated patients had better results than the tracheostomy group, but this difference was not significant. Individuals in the control group had significantly higher Body Cathexis Scales scores than individuals in the tracheostomy and post-operative groups. Long-term tracheostomy negatively affected this scale (48.4 vs. 7.4 months; $\mathrm{P}=0.06$ ).

Hashmi et al. (14) conducted a combined prospective and retrospective cohort study using the SF-12 survey. The study comprised individuals with benign and malignant diseases and concluded that a planned tracheostomy resulted in an improvement in the physical health and the ability to perform activities of daily living. Nevertheless, there was a decline in the mental health, probably due to worsening of self-esteem. These results could be explained by the fact that patients that were submitted to tracheostomy had severe laryngeal obstruction, and were already physically impaired. Thus, tracheostomy was beneficial and actually improved the HQL. 
SF-12 component summary scores are comparable to SF36 scores, since one is derived from the other. If having only adequate physical and mental health summary scores is of interest, the SF12 is an interesting tool, because it is faster to fill out and is as reliable as the SF-36. The Body Cathexis Scale was the first psychometric instrument to measure body dissatisfaction. It provides an objective measure of the individuals' feelings towards various impressions of the body. Therefore, it could be a useful tool to measure the impact of a tracheostomy on the physical aspects of a patient, but it does not have the capability to analyze mental and psychological impairment. Our results with SF-36 suggest that tracheostomy severely affects quality of life, physically and mentally.

The present study has limitations. All participants were recruited at a single institution. That can potentially reduce the generalizability of the study. In addition, the SF-36 questionnaire was only applied once. Moreover, the mean treatment time was short (4.4 months) and this could have a potential impact on the results. On the other hand, the study population was more homogeneous because only patients with benign post-intubation tracheal stenosis were included. The selection bias was minimized by the use of a strict protocol for patient enrollment. Other factors such as socioeconomic status, education level, unemployment, poor social and family support, addiction and depression, were not taken into account. Nonetheless, we believe that these issues were partially dealt with in the random selection of the cohort.

Regardless of the limitations, this study opens a new window in the assessment of patients with tracheal stenosis using a validated and available tool such as the SF-36 questionnaire. This has been particularly important in our academic referral center for tracheal and airway diseases, where we have witnessed an increasing number of complex tracheal and laryngotracheal stenosis. We are currently expanding this study locally and hopefully into a multicenter trial soon in order to collect a larger data sample.

\section{Conclusions}

In conclusion, the overall quality of life of patients with tracheal stenosis treated with stents or tracheostomy is severely impaired. Physical and mental discomfort is attenuated by total treatment time.

\section{Acknowledgements}

None.

\section{Footnote}

Conflicts of Interest: The authors have no conflicts of interest to declare.

Ethical Statement: The study was approved by the local Institutional Review Board (Cappesq \#719897). Date of approval: February $12^{\text {th }}, 2014$.

\section{References}

1. Hagmeyer L, Oesterlee U, Treml M, et al. Successful weaning and decannulation after interventional bronchoscopic recanalization of tracheal stenosis. J Crit Care 2014;29:695.e9-14.

2. Bibas BJ, Terra RM, Oliveira Junior AL, et al. Predictors for postoperative complications after tracheal resection. Ann Thorac Surg 2014;98:277-82.

3. Terra RM, Bibas BJ, Minamoto H, et al. Decannulation in tracheal stenosis deemed inoperable is possible after longterm airway stenting. Ann Thorac Surg 2013;95:440-4.

4. Soto M, Failde I, Márquez S, et al. Physical and mental component summaries score of the SF-36 in coronary patients. Qual Life Res 2005;14:759-68.

5. Ware JE, Snow KK, Kosinski M, et al. SF-36 Health Survey: Manual and Interpretation Guide. Boston, MA: Health Institute, New England Medical Center, 1994.

6. Bibas BJ, Bibas RA. A new technique for T-tube insertion in tracheal stenosis located above the tracheal stoma. Ann Thorac Surg 2005;80:2387-9.

7. Ciconelli R, Ferraz M, Santos W, et al. Tradução para a língua portuguesa e validação do questionário genérico de avaliação de qualidade de vida SF-36 (Brasil SF-36). Rev Bras Reumatol 1999;39:143-50.

8. World Medical Association. World Medical Association Declaration of Helsinki: ethical principles for medical research involving human subjects. JAMA 2013;310:2191-4.

9. Terra RM, Minamoto H, Tedde ML, et al. Self-expanding stent made of polyester mesh with silicon coating (Polyflex) in the treatment of inoperable tracheal stenoses. J Bras Pneumol 2007;33:241-7.

10. Terra RM, Minamoto H, Carneiro F, et al. Laryngeal split and rib cartilage interpositional grafting: treatment option for glottic/subglottic stenosis in adults. J Thorac Cardiovasc Surg 2009;137:818-23.

11. Bibas BJ, Guerreiro Cardoso PF, Minamoto H, et al. Surgical Management of Benign Acquired Tracheoesophageal Fistulas: A Ten-Year Experience. Ann 
Thorac Surg 2016;102:1081-7.

12. Bibas BJ, Cardoso PF, Minamoto H, et al. Surgery for intrathoracic tracheoesophageal and bronchoesophageal fistula. Ann Transl Med 2018;6:210.

13. Gilony D, Gilboa D, Blumstein T, et al. Effects of

Cite this article as: Bibas BJ, Cardoso PF, Salati M, Minamoto H, Tamagno MF, Terra RM, Pêgo-Fernandes PM. Healthrelated quality of life evaluation in patients with non-surgical benign tracheal stenosis. J Thorac Dis 2018;10(8):4782-4788. doi: $10.21037 /$ jtd.2018.07.80 tracheostomy on well-being and body-image perceptions. Otolaryngol Head Neck Surg 2005;133:366-71.

14. Hashmi NK, Ransom E, Nardone H, et al. Quality of life and self-image in patients undergoing tracheostomy. Laryngoscope 2010;120 Suppl 4:S196. 